\title{
Group-Selection for 'Goodness'? An Account of Chickens, a Comparison with Plants, and Implications for Humans
}

\author{
Lonnie W. Aarssen*
}

Department of Biology, Queen's University, Kingston, ON, K7L 3N6, Canada

\begin{abstract}
Can 'goodness' evolve in humans through group-selection? It can, according to a new book, Evolution For Everyone, which claims support for this possibility from a recent research program involving artificial group-selection in chickens. Data from this study, showing increased egg production across generations, are interpreted as a product of the evolution of good, cooperative behaviour among hens. In this commentary, I propose that there is a more parsimonious and more plausible interpretation for these results involving something much less noble - a system of dominance and subordination, where maximization of egg production across generations resulted from selection that increased the relative frequency of subordinate 'crumb-collector' hens that passively tolerate domination by relatively few aggressive 'strongpluckers'. Evidence for such dominance/suppression effects in maximizing group productivity is common in vegetation where most coexisting plants are relatively small and highly suppressed by a few larger ones, and where high productivity is interpreted by plant ecologists, not in terms of any group-selection effects, but rather in terms of traditional individual Darwinian natural selection favouring tolerance of resource deprivation, reproductive economy, and complementary resource use strategies.
\end{abstract}

A recent research program involving artificial selection for egg production in caged hens [1-5] has been regarded [e.g. 6] as one of the most convincing examples of empirical evidence for the possibility of group-selection in nature. In a new book, "Evolution For Everyone" [7], this study is proposed as a showcase example for considering how "goodness can evolve" in humans (p. 31), involving jointly beneficial outcomes of natural selection operating at the level of groups. 'Good' hens, like 'good' people, are defined in this context by traits that promote living together in peaceful, productive groups.

The chicken study tested two methods for increasing egg production. The above book [7] describes them (p. 34): "The first method involved selecting the most productive hen from each of a number of cages to breed the next generation of hens. The second method involved selecting all the hens from the most productive cages to breed the next generation of hens". After six generations, a cage of hens resulting from the first method is described as follows: "Inside the cage were only three hens, not nine, because the other six hens had been murdered. The three survivors had plucked each other during their incessant attacks and were now nearly featherless. Egg production plummeted during the course of the experiment, even though the most productive individuals had been selected each and every generation. ... The most productive individuals had achieved their success by suppressing the productivity of their cage mates". In cages resulting from the second method, however, all hens survived and were fully feathered; "Egg production had increased dramatically during the course of the experiment. By selecting whole groups, .. [this] .. selected against aggressive traits

*Address correspondence to this author at the Department of Biology, Queen's University, Kingston, ON, K7L 3N6, Canada;

E-mail: aarssenl@biology.queensu.ca and for cooperative traits that enabled hens to coexist harmoniously".

In this commentary, I do not challenge the claim that altruism or 'goodness' can evolve through group selection. Rather, I challenge the claim that this particular study of chickens provides convincing evidence for this possibility. Groups - distinguished by variation in total egg production were indeed selected intentionally by researchers in this study, and this resulted in changes in genotypic composition of hens across generations. But, as I will argue below, it is more likely that variation in total egg production, rather than a product of variation in the level of 'goodness' between interacting hens, was more proximately a consequence of variation in the egg production contributed by just one particular component of the group. Hence, although the methodology involved selecting groups, researchers were effectively imposing traditional, individual selection, without knowing it, fortuitously in favour of just this one component of the group. While the outcome appeared, therefore, to involve the evolution of 'good' and 'cooperative' group behaviour, the results are instead more likely to be a product of the generation of something altogether different.

My interpretation can be illustrated by considering parallel effects that are evident from research in plant ecology. In vegetation, assemblies of certain plant species living together can often be shown to out-yield vegetation comprised of just the single most productive species [8] - an effect that is also evident from inter-cropping in agriculture [9]. In natural vegetation, when changes in species or genotype composition over time lead to increases in total group productivity, plant ecologists never interpret this as a consequence of group-selection involving greater group cooperation. Typically, if selection is involved at all, it is regarded as a product of individual natural selection involving 'complementarity' [8]. 
Complementarity is associated with two effects: The first involves 'niche differentiation' between different types (species or genotypes of plants or hens). This results when a single type fails to possess all of the traits that are required to use all of the available resources. Hence, after intense competition between individuals that are all of one type, typically there are some resources still left unused, and so productivity at the whole group level is limited. A greater variety of types then, as in a cage of hens with different foraging strategies, is likely to use available food resources more completely, thus allowing for the possibility of higher group productivity (egg production).

The second effect results when it is possible to have reproductive success under competition without being a particularly aggressive competitor. This is common in plant communities, where larger or faster growing plants obtain more resources, but many smaller ones commonly coexist with them by using resources that are left over. In other words, the smaller ones are adapted to tolerate resource deprivation; i.e. they can reproduce with fewer resources, and so have smaller 'physical-space-niches' [10]. Similarly, in cages of mixed hen types, 'strong-pluckers' can be expected to get the most resources as individuals, while more passive 'crumb-collectors' will obtain resources that strong-pluckers leave behind. Crumb-collectors obviously do not have high individual productivity, but they are well equipped to survive resource deprivation from competition, and avoid the impact of strong-pluckers. Presumably, this results because crumbcollectors do not 'pluck back', and so strong-pluckers leave them alone much of the time. Although suppressed by competition, crumb-collectors nevertheless manage at least some egg production. These subordinate hens, like many subordinate plants within vegetation, have 'reproductive economy' an ability to produce at least some offspring despite severe resource deprivation [11].

Complementarity, therefore, can explain the higher total egg production in cages of chickens belonging to a variety of behavioral types, compared with cages in which all individuals are bred from only one type. Because strong-pluckers are intrinsically aggressive, they are intrinsically discontent to be mere crumb-collectors; apparently they will pluck to the death rather than settle for 'crumbs'. Hence, when all of the resident hens within a cage are strong-pluckers, despite their impressive individual efforts, as a monotypic group they are not equipped to use all of the available resources and translate them into egg production. Crumb-collectors, in contrast of course, are content with 'crumbs'. Their success lies primarily in rejecting the challenge from strong-pluckers, and instead, diverting or fleeing from their attacks while furtively grasping a few morsels along the way. Accordingly, each crumb-collector gathers only enough to support a relatively meager clutch of eggs, but the collective production of several resident crumb-collectors contributes significantly to the group total.

What does this have to do with evolution by 'group selection'? Nothing in my view. What then accounts for the increasing egg production across generations in this study of chickens? The answer, I propose, is that by selecting the most productive groups (cages) in the each generation, researchers in this study were inadvertently selecting cages that just had higher and higher relative frequencies of crumb- collectors. This ensured that more and more of the available resources were being used up in each successive generation, thus maximizing collective egg production. In other words, the researchers were, in effect, imposing individual selection in favour of the reproductive success of only one component of the group - the crumb-collectors.

Importantly, this selection in favour of crumb collectors was achieved without disfavouring the reproductive success of resident strong-pluckers, because of the complementarity between these two strategies. In other words, the reproductive success of resident strong-pluckers in each generation was limited, I suggest, not by the growing representation of crumb-collectors, but instead by other resident strongpluckers, which were busy plucking each other while the crumb-collectors were scurrying about, largely unnoticed, gathering crumbs. Hence, in the 'group-selection' method of this study, the strong-pluckers in each generation were limiting the egg production of other resident hens (as they are well equipped to do), but mostly they were just limiting other strong-pluckers - just as they were doing in the first selection method, where only strong-pluckers were allowed to breed. Simply put, the additional crumb-collectors that accumulated across generations were mainly just using resources that the strong-pluckers in each generation weren't going to be using anyway; i.e. they had 'niche differentiation'. In each generation of this selection, we can expect that the most dominant strong-plucker in residence always had the highest individual egg production, but much or perhaps most of the total group egg production was probably a result of the collective contribution from the more numerous subordinate crumb-collectors. There is no reason to surmise that either of these individual strategies was altered by this selection, or that any new cooperative 'group' strategy evolved as a consequence of selection.

As the maximum possible egg production is approached in the above process, it seems reasonable to predict, therefore, that crumb-collectors should virtually always outnumber strong-pluckers. And if there is any potential for increased egg production, this would be more likely to happen by adding another crumb-collector than by adding another strong-plucker. In other words, if dominants are already present, it generally pays more to be a subordinate, and we would expect this to be associated with an evolutionarily stable proportion of dominants and subordinates. Moreover, rather than just two distinct types, perhaps there is a stable distribution involving a continuum of tactics between two extreme strategies, ranging from highly aggressive pluckers to highly efficient crumb-collectors.

Similar features characterize the assembly of natural plant communities, where there is usually a wide range of plant sizes among coexisting species, but where the vast majority are relatively small, in virtually every habitat type on earth [10]. Moreover, even within many natural populations of a single plant species, the vast majority of resident reproductive plants are also relatively small. In fact, most of the total offspring production for the next generation within many plant populations typically comes from the collective output of the numerous resident plants belonging to relatively small size classes, and this is especially true under the most crowded conditions, where competition intensity is severe [12]. 
Both very large and very small plant sizes, therefore, are associated with effective coexisting strategies for leaving descendants under intense competition in vegetation, but more often, selection has favoured individual strategies that involve relatively small, subordinate plants. Similarly, both strong-pluckers and crumb-collectors in hens can be viewed as effective alternative coexisting strategies that have each evolved by individual natural selection. And when strongpluckers are present, opportunities for increased egg production depend primarily on opportunities for an increased representation of subordinate crumb-collectors, through their individual natural selection.

In conclusion, I propose that the artificial researcherimposed group-selection on these chickens served only to illustrate what we would predict should happen naturally over time (albeit more slowly) in any population that contains a mixture of foraging strategies - i.e., a gradual increase in egg production as a product of individual natural selection, associated with a progression toward an evolutionarily stable proportion of strong-pluckers and crumbcollectors. Rather than suggesting that "goodness can evolve" in hens, or in humans [7], therefore, the growing collective egg production across generations in this chicken study is more analogous to the growing human population size that results when poor human 'crumb-collectors' accumulate in the squalor of slums in human societies that are dominated by relatively few, rich 'strong-pluckers'.

\section{ACKNOWLEDGEMENTS}

W.M. Muir provided the references to the original articles reporting the results of the chicken study described by Wilson (2007). Amber Budden and Tom Tregenza provided helpful comments on the manuscript.

\section{REFERENCES}

[1] Craig, .JV.; Muir, W.M. Group selection for adaptation to multiplehen cages: Beak-related mortality, feathering and body weight responses. Poultry Sci., 1996, 75: 294-302.

[2] Muir, W.M. Group selection for adaptations to multiple-hen cages: selection program and direct responses. Poultry Sci., 1996, 75: $447-$ 458.

[3] Muir, W.M. Indirect selection for improvement of animal wellbeing. In Poultry Genetics, Breeding and Biotechnology; Muir W.M.; Aggrey S., Eds.; CABI Press: Cambridge, MA, 2003; pp. 247-256.

[4] Muir, W.M.; Craig, J.V. Improving animal well-being through genetic selection. Poultry Sci. 1998, 77: 1781-1788.

[5] Muir, W.M.; Cheng, H. Breeding for productivity and welfare. In Welfare of the Laying Hen: Poultry Science Symposium Series, No. 27; Perry, G., Ed.; CABI Press: Cambridge, MA, 2004; pp. 123138.

[6] Goodnight, C.J.; Stevens, L. Experimental studies of group selection: What do they tell us about group selection in nature? Am. Nat., 1997, 150: S59-S79.

[7] Wilson, D.S. Evolution For Everyone. Bantom Dell: New York, 2007.

[8] Loreau, M.; Naeem, S.; Inchausti, P., Eds. Biodiversity and Ecosystem Functioning: Synthesis and Perspectives. Oxford University Press: Oxford, 2002

[9] Swift, M.J.; Anderson, J.M. Biodiversity and ecosystem function in agricultural systems. In Biodiversity and ecosystem function; Schulze, E.-D.; Mooney, H.A., Eds.; Springer-Verlag: Berlin, 1994.

[10] Aarssen, L.W.; Schamp, B.S.; Pither, J. Why are there so many small plants? Implications for species coexistence. J. Ecol., 2006 94: 569-580.

[11] Aarssen, L.W. Death without sex - the problem of small and selection for reproductive economy in flowering plants. Evol. Ecol., 2008, 22: 279-298.

[12] Chambers, J.; Aarssen, L.W. Offspring for the next generation: most are produced by small plants within herbaceous populations. Evol. Ecol., 2008, (in press). 